\title{
Serum miR-27a is a biomarker for the prognosis of non-small cell lung cancer patients receiving chemotherapy
}

\author{
Erfu Xie ${ }^{1,2}$, Mingxin Lin ${ }^{1,2}$, Ziwei Sun ${ }^{1,2}$, Yuexinzi Jin ${ }^{1,2}$, Shichang Zhang ${ }^{1,2}$, Lei Huang ${ }^{1,2}$, Ruihong Sun ${ }^{1,2}$, \\ Fang Wang ${ }^{1,2}$, Shiyang Pan ${ }^{1,2}$ \\ ${ }^{1}$ Department of Laboratory Medicine, the First Affiliated Hospital of Nanjing Medical University, Nanjing, China; ${ }^{2}$ National Key Clinical \\ Department of Laboratory Medicine, Nanjing, China \\ Contributions: (I) Conception and design: E Xie, S Pan; (II) Administrative support: F Wang, S Pan, S Zhang; (III) Provision of study materials or \\ patients: Z Sun, L Huang, R Sun; (IV) Collection and assembly of data: E Xie, M Lin, Z Sun; (V) Data analysis and interpretation: All authors; (VI) \\ Manuscript writing: All authors; (VII) Final approval of manuscript: All authors. \\ Correspondence to: Shiyang Pan. Department of Laboratory Medicine, the First Affiliated Hospital of Nanjing Medical University, Guangzhou Road \\ No. 300, Nanjing 210029, China. Email: sypan@njmu.edu.cn.
}

Background: Lung cancer has a high incidence and a 5 -year survival rate of less than $15 \%$. Nonsmall cell lung cancer (NSCLC) accounts for approximately $85 \%$ of lung cancer cases. Chemotherapy and immunotherapy are the most frequently used alternative treatments for patients with advancedstage NSCLC in whom surgery failed. Previous studies have suggested that miR-27a is involved in cancer development and progression. The purpose of this study was to investigate the clinical value of miR-27a in the prognosis of NSCLC patients after chemotherapy.

Methods: Flow cytometry was used to detect the apoptosis rate of SPC-A1 cells treated with optical cisplatin at different times. Simultaneously, the expression of miR-27a in supernatants and cells was detected. Fifty-two newly diagnosed NSCLC patients were recruited. All patients received gemcitabine and cisplatin as first-line chemotherapy and docetaxel as second-line chemotherapy. At the end of every chemotherapy cycle, a therapeutic evaluation was performed according to the RECIST criteria. The expression of serum miR-27a was detected in each cycle.

Results: After treatment with $2.5 \mu \mathrm{g} / \mathrm{mL}$ cisplatin, the apoptosis rates of SPC-A1 cells were significantly greater than those of the paired untreated control groups at 12, 24, 48 and $72 \mathrm{~h}$. The expression of miR$27 \mathrm{a}$ in supernatants and cells was also consistent with the apoptosis rate and changed a time-dependent manner. The chi-square test showed that an increase in miR-27a after chemotherapy was more common in patients who achieved partial response (PR) than in those who achieved no response (NR) $(61.5 \%$ vs. $30.8 \%$, $\mathrm{P}=0.026)$. Kaplan-Meier survival analysis indicated that patients with decreased miR-27a levels had poorer outcomes than those with increased miR-27a levels $(\mathrm{P}<0.05)$. Furthermore, dynamic changes in serum miR27 a with a gradual increasing trend during chemotherapy predicted a good prognosis.

Conclusions: Collectively, our results suggest that miR-27a is involved in the apoptosis of lung cancer cells and that serum miR-27a levels are related to the prognosis of NSCLC patients. The expression levels of miR-27a in the serum may be an independent predictor for the prognosis of NSCLC.

Keywords: Non-small cell lung cancer (NSCLC); miR-27a; prognosis; chemotherapy; individualized treatment

Submitted Nov 16, 2020. Accepted for publication Apr 08, 2021.

doi: $10.21037 /$ tcr-20-3276

View this article at: http://dx.doi.org/10.21037/tcr-20-3276 


\section{Introduction}

Lung cancer affects the respiratory system and has a high incidence and a 5 -year survival rate less than $15 \%$. Nonsmall cell lung cancer (NSCLC) is the most common type of lung cancer and accounts for approximately $85 \%$ of lung cancer cases $(1,2)$. Surgery is the most effective treatment for patients with early-stage NSCLC. However, most patients with advanced-stage lung cancer do not undergo surgery due to the low sensitivity and specificity of diagnostic methods (3). Currently, the most frequently used alternative treatments for NSCLC patients are chemotherapy and immunotherapy, which can help prolong overall survival and improve quality of life (4). Although chemotherapeutic agents, including cisplatin, are able to greatly improve the life quality of patients with advanced NSCLC (5), the prognosis of patients remains unsatisfactory. Thus, it is necessary to identify the specific survival markers of advanced NSCLC, which assist to reflect the therapeutic efficacy of chemotherapeutic agents and the prognosis.

MicroRNAs (miRNAs) are RNAs that are 19-25 nucleotide in length and have been widely recognized as key mediators in the epigenetic control of gene expression at the post-transcriptional level by base pairing to complementary sites on the 3'-untranslated region (3'-UTR) of target messenger RNAs (mRNAs) (6). They play an important regulatory role in a series of biological and pathological processes, such as development, differentiation, proliferation and apoptosis (7-10). Similar to the classification of genes, miRNAs can also be divided into onco-miRNAs and tumour suppressor miRNAs.

Previously, our research identified a monoclonal antibody called NJ001, whose function is specific to NSCLC (11). In this earlier study, we found that some miRNAs, including miR-638 and miR-27a, exhibited the most significant timedependent expression in response to NJ001 (GEO accession number: GSE51947). Subsequently, we reported that the serum miR-638 level may be considered an emerging independent predictor for the prognosis of NSCLC because it is related to the survival of NSCLC patients (12). Moreover, recent publications have demonstrated that miR$27 \mathrm{a}$, as a tumour suppressor miRNA, plays a vital role in the progression of cancers and influences tumourigenesis, tumour cell proliferation, apoptosis, invasion, and migration and angiogenesis (13-16). These results suggest that miR$27 \mathrm{a}$ is involved in the occurrence and progression of cancer. However, previous studies have rarely reported the prognostic value of miR-27a in advanced NSCLC. In this study, we identified the prognostic potential of miR-27a, which provides a theoretical foundation for the clinical application of miR-27a in NSCLC.

We present the following article in accordance with the STROBE reporting checklist (available at http://dx.doi. org/10.21037/tcr-20-3276).

\section{Methods}

\section{Collection of cultured supernatants and SPC-A1 cells for in vitro experiments}

The human lung adenocarcinoma cell line SPC-A1 was purchased from the Cell Bank of Type Culture Collection of the Chinese Academy of Sciences, Shanghai, China. The SPC-A1 cell line was not contaminated with mycoplasma or bacteria according to mass spectrometry results from the European Cell Culture Preservation Center. SPC-A1 cells were plated into six-well plates $\left(1.5 \times 10^{6}\right.$ per well $)$ and treated with complete RPMI 1640 medium alone (as the control group) or $2.5 \mu \mathrm{g} / \mathrm{mL}$ cisplatin and incubated at $37{ }^{\circ} \mathrm{C}$ and $5 \% \mathrm{CO}_{2}$ for $12,24,48$ or $72 \mathrm{~h}$. The optical treatment concentrations for cisplatin were based on our previous research (12). Harvested cultured supernatants and SPC-A1 cells were used for miR-27a detection. The whole experiment was repeated three times, and the mean of three time points were used.

\section{Detection of the apoptosis rates of SPC-A1 cells}

SPC-A1 cells $\left(1 \times 10^{6}\right)$ were collected, and the apoptosis rate was determined by flow cytometry. According to the manufacturer's instructions, SPC-A1 cells were stained with $5 \mu \mathrm{L}$ propidium iodide and $5 \mu \mathrm{L}$ annexin V-FITC (Annexin V-FITC Apoptosis Detection Kit, BD Biosciences, Franklin, NJ, USA). All analyses were performed on a flow cytometer (BD Biosciences).

\section{NSCLC patients and serum specimens}

Fifty-two patients diagnosed with advanced NSCLC between March 2010 and May 2012 at the First Affiliated Hospital of Nanjing Medical University were recruited [32 males and 20 females, aged 31-77 years (median age: 61 years)]. The study was conducted in accordance with the Declaration of Helsinki (as revised in 2013). All patients with newly diagnosed lung adenocarcinoma were unable to undergo radical surgery and did not receive radiotherapy or chemotherapy. The diagnosis of lung cancer was confirmed 
by a histopathological examination. All patients received gemcitabine and cisplatin as first-line chemotherapy and docetaxel as second-line chemotherapy. At the end of every chemotherapy cycle, a therapeutic evaluation was performed according to the RECIST criteria and included complete response (CR), partial response (PR), stable disease (SD) and progressive disease (PD). SD and PD belong to the no response (NR) category. Patients were followed until March 2013. The present study was approved by the Research Ethics Committee of the First Affiliated Hospital of Nanjing Medical University (No. 2015-SRFA-065, Nanjing, China), and all patients provided written consent prior to study enrolment. After obtaining informed consent, blood was drawn before and after chemotherapy. Whole blood was centrifuged at 2,500 $\mathrm{g}$ for $10 \mathrm{~min}$, followed by $16,000 \mathrm{~g}$ for $10 \mathrm{~min}$ to completely remove cell debris. Then, the serum was separated, aliquoted and stored at $-70^{\circ} \mathrm{C}$.

\section{RNA extraction}

According to the manufacturer's protocol, total cell supernatants and serum RNA were extracted for quantitative RT-PCR (qRT-PCR) using the miRNeasy Mini Kit (QIAgen, Düsseldorf, Germany). cel-miR-39 was added as an internal control (59-ucaccggguguaaaucagcuug-39; final concentration: $10^{-5} \mathrm{pmol} / \mathrm{mL}$ ). Total RNA was extracted from cells using QIAzol Lysis Reagent (QIAgen, Düsseldorf, Germany) according to the manufacturer's protocol. A UV spectrophotometer (at 260 and $280 \mathrm{~nm}$ ) and agarose gel electrophoresis were used to validate the RNA concentration and quality.

\section{qRT-PCR}

The miR-27a expression levels in the supernatants, cells and serum were detected by qRT-PCR. According to the manufacturer's instructions, $5.84 \mu \mathrm{L}$ of reverse transcription (RT) reagent (Applied Biosystems, Foster City, CA, USA), including stem-loop RT primers, was mixed with $9.16 \mu \mathrm{L}$ of RNA in each reaction. RT reactions proceeded as follows: $16{ }^{\circ} \mathrm{C}$ for $30 \mathrm{~min}$, followed by $42{ }^{\circ} \mathrm{C}$ for $30 \mathrm{~min}$, $85^{\circ} \mathrm{C}$ for $5 \mathrm{~min}$ and then maintained at $4{ }^{\circ} \mathrm{C}$. Then, PCR was performed on the ABI 7500 real-time system as follows: 10 min at $95{ }^{\circ} \mathrm{C}, 15 \mathrm{~s}$ at $95^{\circ} \mathrm{C}$ with 45 cycles and $1 \mathrm{~min}$ at $60^{\circ} \mathrm{C}$. The expression level of miR-27a in the serum is expressed as multiple changes based on the normalized expression level of cel-miR-39. The $2^{-\Delta \mathrm{Ct}}$ formula was used, in which $\Delta \mathrm{Ct}=\mathrm{Ct}_{\mathrm{miR}-27 \mathrm{a}}-\mathrm{Ct}_{\mathrm{miR}-39}$. The expression level of $\mathrm{miR}-27 \mathrm{a}$ in the supernatants is expressed as multiple changes based on the normalized expression level of celmiR-39. The $2^{-\Delta \Delta C t}$ formula was used, in which $\Delta \Delta C t=$ $\left(\mathrm{Ct}_{\text {miR-27a }}-\mathrm{Ct}_{\text {miR-39 }}\right)_{\text {treated }}-\left(\mathrm{Ct}_{\text {miR-27a }}-\mathrm{Ct}_{\text {miR-39 }}\right)_{\text {untreated. }}$ The expression level of miR-27a in cells is expressed as multiple changes based on the normalized expression level of U6 snRNA. The $2^{-\Delta \Delta \mathrm{Ct}}$ formula was used, in which $\Delta \Delta \mathrm{Ct}=$ $\left(\mathrm{Ct}_{\text {miR-27a }}-\mathrm{Ct}_{\mathrm{U} 6}\right)_{\text {treated }}-\left(\mathrm{Ct}_{\text {miR-27a }}-\mathrm{Ct}_{\mathrm{U} 6}\right)_{\text {untreated }}$.

\section{Statistical analysis}

SPSS 16.0 software (Chicago, IL, USA) and GraphPad Prism 7.0 (San Diego, CA, USA) were used for statistical analyses. The proportions of miR-27a expression levels altered before and after chemotherapy between the PR and NR (including PD and SD) populations were compared using the chi-square test. The Kaplan-Meier method was used for survival analysis. We compared survival times between groups with the log-rank test. A P value $<0.05$ was considered statistically significant.

\section{Results}

\section{Apoptosis of SPC-A1 cells induced by cisplatin}

SPC-A1 cells were treated with $2.5 \mu \mathrm{g} / \mathrm{mL}$ cisplatin at different times to evaluate the effect of cisplatin on apoptosis. Flow cytometry was used to detect the apoptosis rate at 12, 24, 48 and $72 \mathrm{~h}$ after treatment with or without cisplatin. Compared with the paired untreated control cells, the morphology of SPC-A1 cells was changed (Figure 1). Moreover, the apoptosis rates of the SPC-A1 cells cultured for 12, 24, 48 and $72 \mathrm{~h}$ with cisplatin were significantly higher than those without cisplatin (Figures 2,3). With prolonged cisplatin exposure, these apoptosis rates increased more markedly. These results suggest that cisplatin induces apoptosis in SPC-A1 cells in a time-dependent manner.

\section{miR-27a expression in the supernatant of SPC-A1 cells increased in a time-dependent manner after cisplatin treatment}

qRT-PCR was used to detect the expression level of miR$27 \mathrm{a}$ in the supernatant of SPC-A1 cells treated with cisplatin. We found that the miR-27a levels in the SPC-A1 cultured supernatants after treatment with $2.5 \mu \mathrm{g} / \mathrm{mL}$ cisplatin for $72 \mathrm{~h}$ were more than four-fold higher than those without treatment (Figure 4A). miR-27a expression 

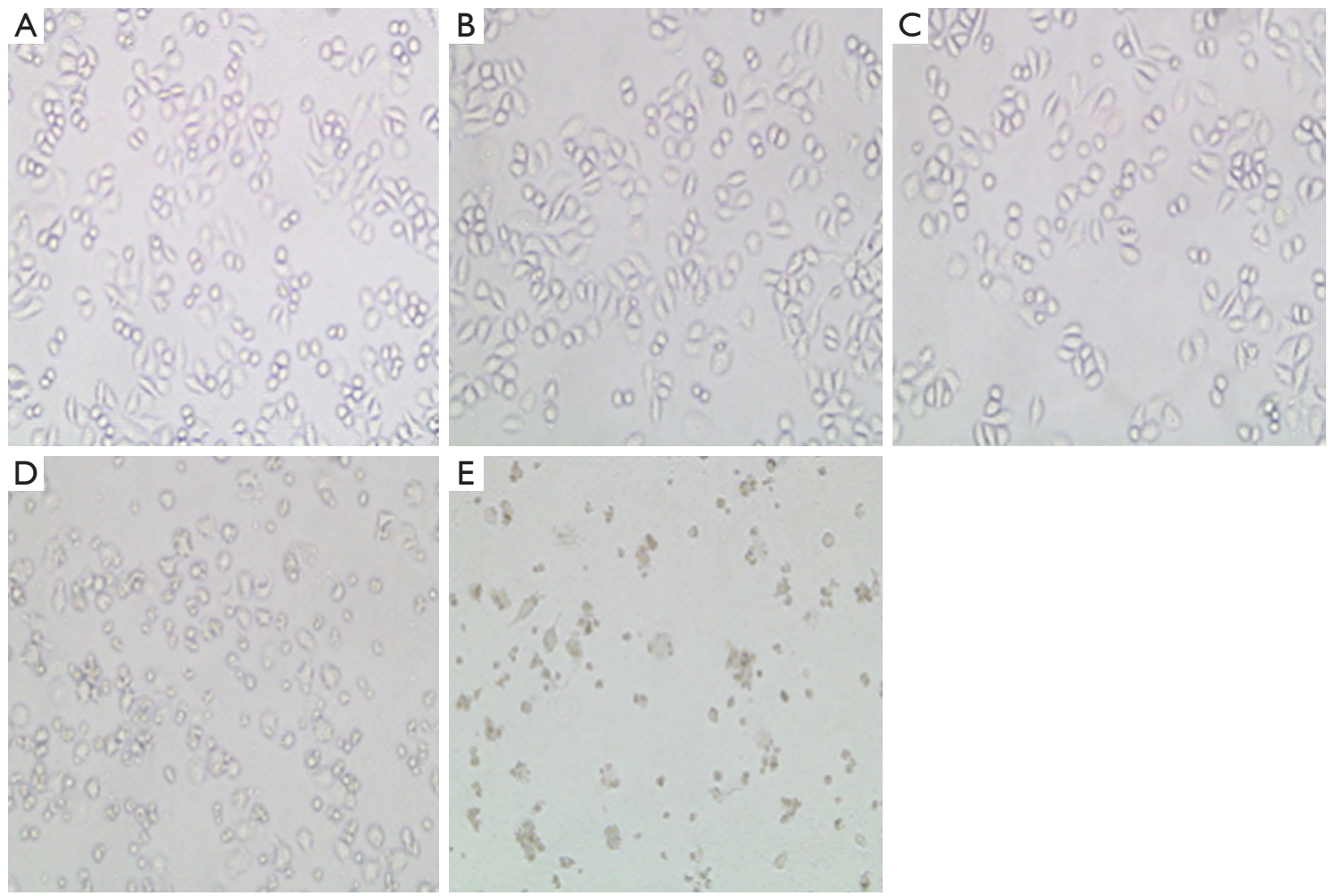

Figure 1 Morphological changes in SPC-A1 cells treated with $2.5 \mu \mathrm{g} / \mathrm{mL}$ cisplatin were observed under a microscope (magnification, $\times 200$ ). (A) Blank control; (B) $12 \mathrm{~h}$; (C) $24 \mathrm{~h}$; (D) 48 h; (E) $72 \mathrm{~h}$.

levels in the treated group $(2.5 \mu \mathrm{g} / \mathrm{mL}$ cisplatin) increased gradually at 12, 24, 48 and $72 \mathrm{~h}$, and the difference between any period of the two groups was significant $(\mathrm{P}<0.05)$. When $2.5 \mu \mathrm{g} / \mathrm{mL}$ cisplatin was used for treatment for different time periods (12-72 h), the levels of miR-27a in supernatants increased in a time-dependent manner (Figure 4A).

\section{miR-27a expression in SPC-A1 cells increased in a time- dependent manner after cisplatin treatment}

qRT-PCR was used to determine the expression levels of miR-27a in SPC-A1 cells treated with cisplatin. The expression levels of miR-27a in SPC-A1 cells treated with $2.5 \mu \mathrm{g} / \mathrm{mL}$ cisplatin for $72 \mathrm{~h}$ were more than 8 times higher than those without cisplatin (Figure 4B). The miR27 a expression levels of SPC-A1 cells in the cisplatin $(2.5 \mu \mathrm{g} / \mathrm{mL})$ group increased between 12 and $24 \mathrm{~h}$, and the miR-27a expression levels between the two groups were significantly different $(\mathrm{P}<0.05)$. However, there was no significant change at 24,48 or $72 \mathrm{~h}$. This result showed that the miR-27a expression levels in the cisplatin $(2.5 \mu \mathrm{g} / \mathrm{mL})$ group entered a platform after $24 \mathrm{~h}$ (Figure $4 B$ ).

Correlation between prognosis and the change in miR-27a expression levels before and after chemotherapy in NSCLC patients

As demonstrated in this study, accompanying the apoptosis of SPC-A1 cells, miR-27a expression levels also increased in SPC-A1 culture supernatants in a time-dependent manner after cisplatin treatment. Therefore, it is necessary to assess the prognostic value of NSCLC patients who receive firstline chemotherapy. The miR-27a expression levels in the sera of 52 patients receiving first-line chemotherapy were monitored before and after chemotherapy. The changes in miR-27a expression levels included an increase in 24 patients and a decrease in 28 patients. The follow-up was conducted until March 2013. The outcomes of the patients included 26 patients with PR and 26 patients with NR (including 10 patients with SD and 16 patients with PD). The detailed information is shown in Table 1. The chisquare test showed that a significant elevation of miR-27a after chemotherapy was more common in patients who 
Control
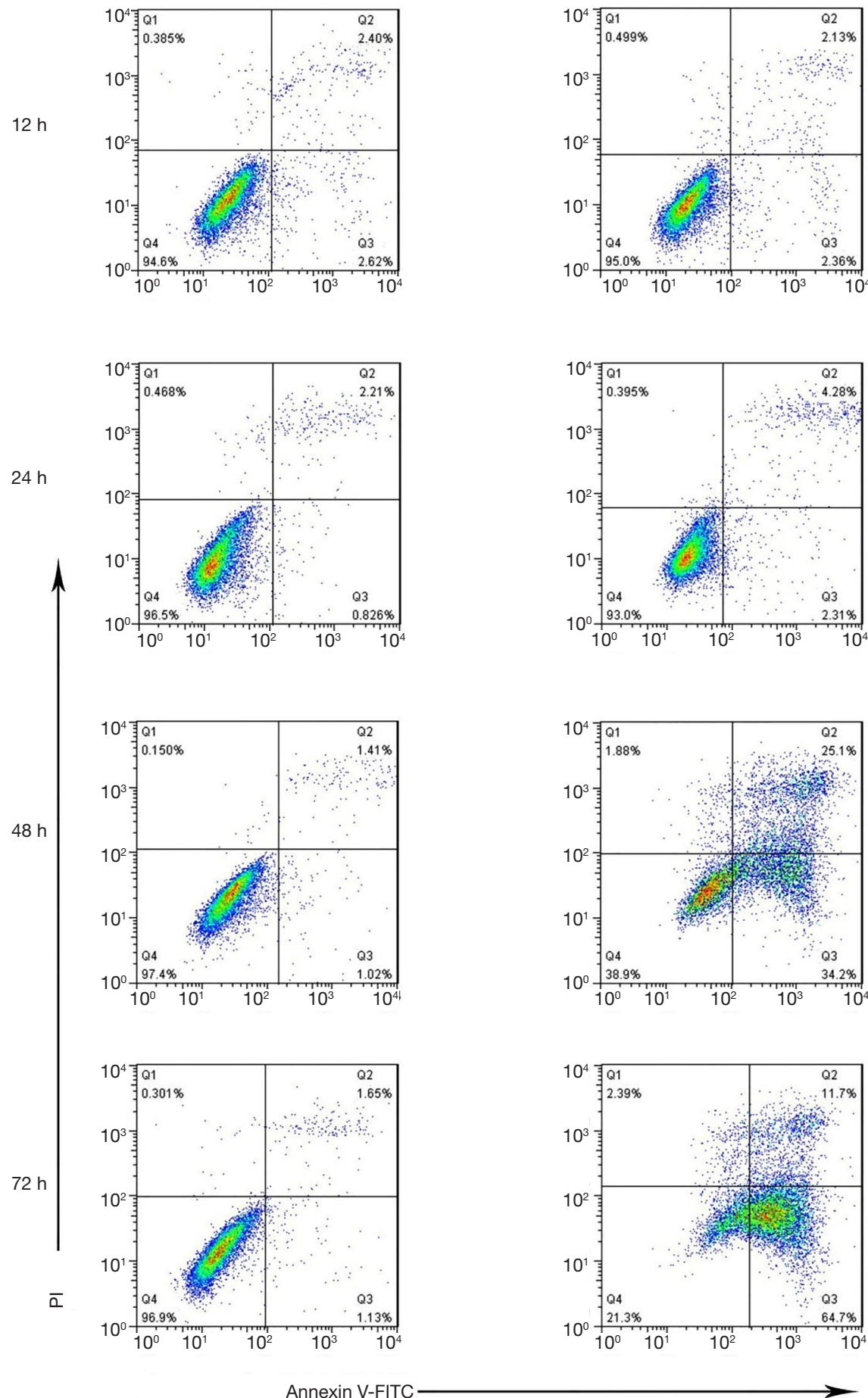

Figure 2 Original flow cytometry plot of SPC-A1 cells at different time points post-treatment with $2.5 \mu \mathrm{g} / \mathrm{mL}$ cisplatin. 


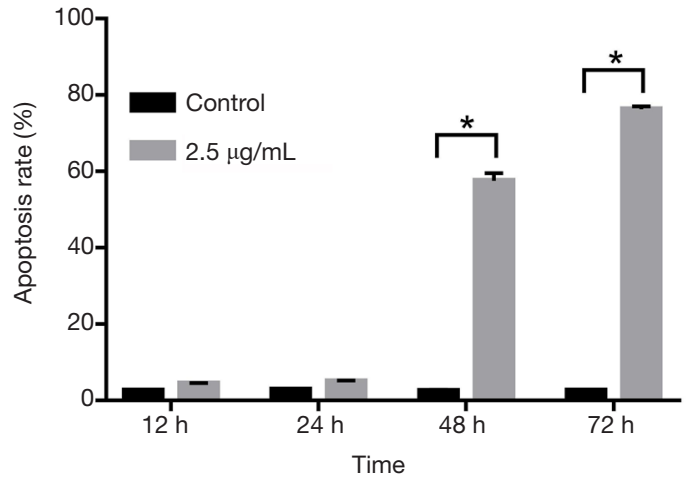

Figure 3 Apoptosis rates of SPC-A1 cells at different time points post-treatment with $2.5 \mu \mathrm{g} / \mathrm{mL}$ cisplatin $\left(^{*}, \mathrm{P}<0.05\right)$. Apoptosis rates of control groups are $2.63 \pm 0.13,2.87 \pm 0.10,2.53 \pm 0.27$, $2.65 \pm 0.07$ after $12,24,48$ and $72 \mathrm{~h}$, respectively. Apoptosis rates of treatment groups are $4.47 \pm 0.11,5.03 \pm 0.17,57.53 \pm 1.99,76.29 \pm 0.79$ $\left({ }^{*}, \mathrm{P}<0.05\right)$.
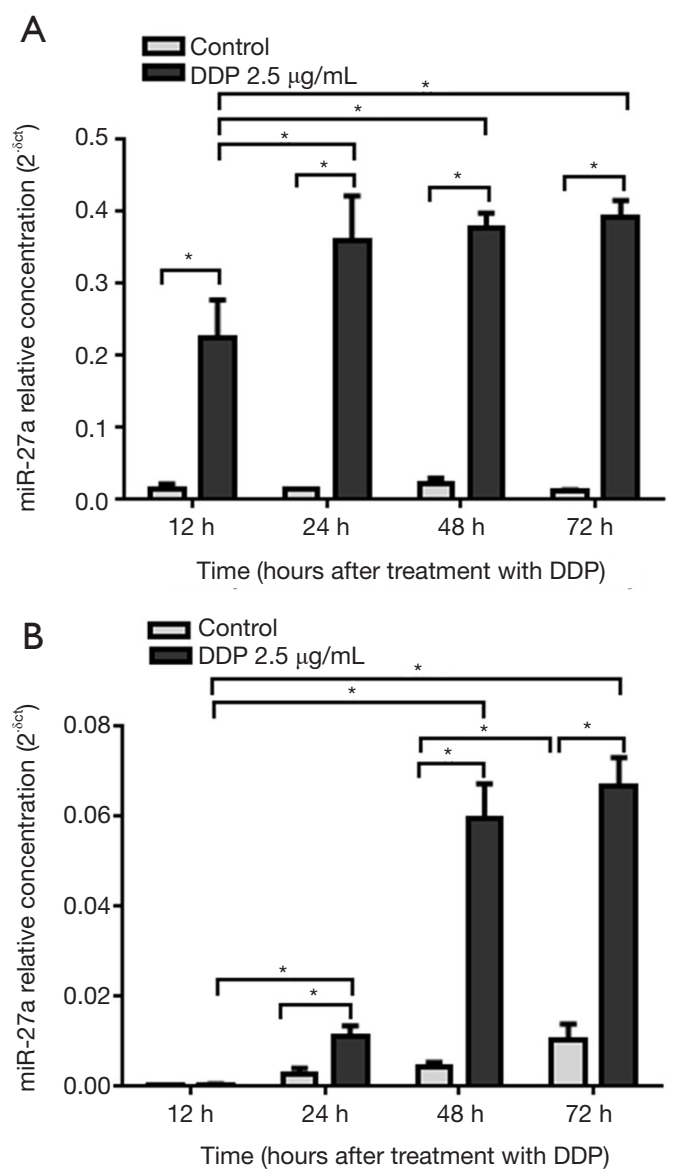

Figure 4 Relative levels of miR-27a expression in SPC-A1 cultured supernatants and cells treated with $2.5 \mu \mathrm{g} / \mathrm{mL}$ cisplatin. (A) Cultured supernatants; (B) SPC-A1 cells (*, P<0.05). achieved PR than in patients who achieved NR $(61.5 \%$ vs. $30.8 \%, \mathrm{P}=0.026)$. These results showed that the increase in the serum miR-27a levels after chemotherapy was related to a good curative effect of NSCLC patients.

\section{Correlation between miR-27a levels and NSCLC patient survival}

At the end of follow-up, 52 patients who completed firstline chemotherapy were divided into two groups according to the increase or decrease in serum miR-27a after chemotherapy. Among them, the serum miR-27a levels of 24 patients were increased, and those of 28 patients were decreased. Kaplan-Meier survival analysis showed that the average survival time of 24 patients with increased serum miR-27a after first-line chemotherapy was 28.4 months, while that of 28 patients with decreased serum miR-27a was 9 months. The survival time in the miR-27a increased group was significantly longer than that in the miR-27a decreased group $(\mathrm{P}<0.05)$. As shown in Figure 5 , the results showed that patients with decreased miR-27a levels had poorer prognoses than those with increased miR-27a levels.

\section{Dynamic changes in serum miR-27a levels during chemotherapy}

The dynamic changes in serum miR-27a were monitored in four patients with NSCLC after different chemotherapy cycles. As shown in Figure 6, patient A received three cycles of first-line chemotherapy. Computed tomography (CT) showed that the tumour was significantly reduced, while tumour markers decreased and serum miR-27a levels increased gradually. At the end of follow-up, the patient survived and achieved PR. Patient B received three cycles of first-line chemotherapy, but due to the progression of multiple metastases throughout the body, 10 months later, the relative content of serum miR-27a levels continued to decrease during chemotherapy. After one cycle of firstline chemotherapy, disease progression resulted in a switch to second-line chemotherapy in patient C. After three cycles of second-line chemotherapy, the relative content of serum miR-27a levels continued to increase. By the end of follow-up, the patient achieved PR. Patient D received two cycles of first-line drug chemotherapy, after which his/her condition became stable, and the patient was discharged. After 1 month, the disease progressed, and the relative content of serum miR-27a levels was significantly decreased. Second-line chemotherapy was used instead of first-line 
Table 1 Correlation between prognosis and the change in miR-27a expression levels before and after chemotherapy in lung adenocarcinoma patients $(\mathrm{n}=52)$

\begin{tabular}{lccc}
\hline \multirow{2}{*}{ Prognosis } & \multicolumn{2}{c}{ miR-27a change after chemotherapy } & Decreasing \\
\cline { 2 - 3 } & Increasing & 10 & 0.026 \\
\hline PR & 16 & 18 & \\
\hline
\end{tabular}

PR, partial response; NR, no response; SD, stable disease; PD, progressive disease.

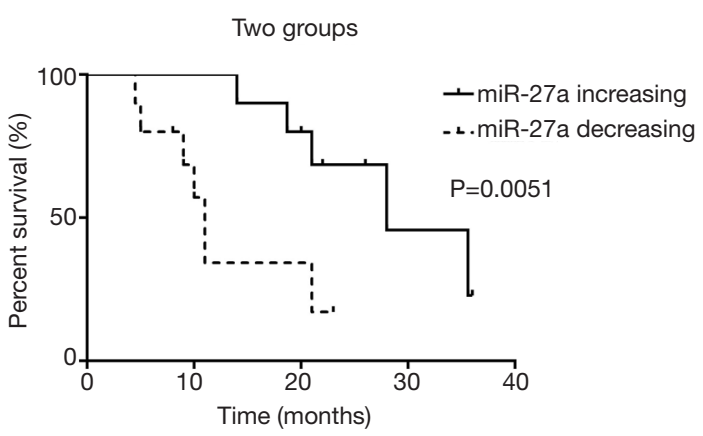

Figure 5 Kaplan-Meier survival curve for the overall survival of NSCLC patients ( $\mathrm{n}=52)$. Kaplan-Meier analysis was performed to evaluate the overall survival of NSCLC patients according to miR27a expression patterns after one cycle of first-line chemotherapy: 24 patients had increasing miR-27a expression levels, and 28 patients had decreasing miR-27a expression levels.

chemotherapy, but the patient died after 11 months. The above four patients also demonstrated that miR-27a can be used as a tumour marker to assess individualized treatment efficacy during chemotherapy.

\section{Discussion}

NSCLC is the most-common subtype of lung cancer, mainly including adenocarcinoma, squamous cell carcinoma and large cell lung cancer (17). Recent studies have found that some novel surgical methods like uniportal videoassisted thoracoscopic surgery and robotic-assisted thoracoscopic surgery have superb advantages over open surgery, which significantly reduces pain, complications, inflammatory response and improves postoperative life quality (18). However, a large proportion of NSCLC patients cannot benefit from these novel surgical methods. An accurate evaluation of the disease condition is conductive to prolong overall survival and effectively reduce the risk of surgical therapies of NSCLC. It is well known that the treatment of NSCLC is stage-specific. Most of patients with advanced NSCLC should be considered for chemotherapy, but their prognosis is still very poor. Therefore, reliable biomarkers are urgently needed for predicting the prognosis of patients with advanced NSCLC receiving chemotherapy.

miRNAs have been investigated for several decades and can negatively regulate gene expression by recognizing the 3'-UTR of a specific target mRNA and inhibiting translation at the post-transcriptional level (19). Recent studies have revealed that miRNAs affect the biological behaviour of lung tumours, and miRNA detection has been widely used for early diagnosis (20), prognosis (21) and therapeutic (22) evaluations, as well as chemosensitivity (23) and chemoresistance (24) evaluations.

Recently, miRNAs have been widely studied as an important means of epigenetic regulation (25). A considerable number of studies have shown that the dysregulation of miRNAs may be involved in the occurrence and development of NSCLC. Therefore, miRNAs could serve as promising molecular markers for clinical application (26). For example, Pan et al. revealed that miR$33 a-5 p$ and miR-128-3p were more sensitive/specific than traditional tumour markers such as NSE, CYFRA21-1 and CA72-4 and could serve as novel biomarkers for the early detection of lung cancer (27). Yang et al. indicated that miR-21 and miR-155 showed promising associations with the prognosis of lung cancer and could predict recurrence and poor survival in NSCLC patients (28). The miR-27 family is composed of miR-27a and miR-27b, and miR-27a plays an important role in tumour development. According to recent studies, miR-27a is significantly dysregulated in various cancers, such as liver cancer (29), NSCLC (30) and prostate cancer (31), and acts as an oncogene. miR27 a also functions as a tumour suppressor in bladder cancer (32), gastric cancer (33), and oesophageal squamous cell cancer (34). These contradictory results have prompted us to investigate the role of miR-27a in tumours and its potential clinical applications. 

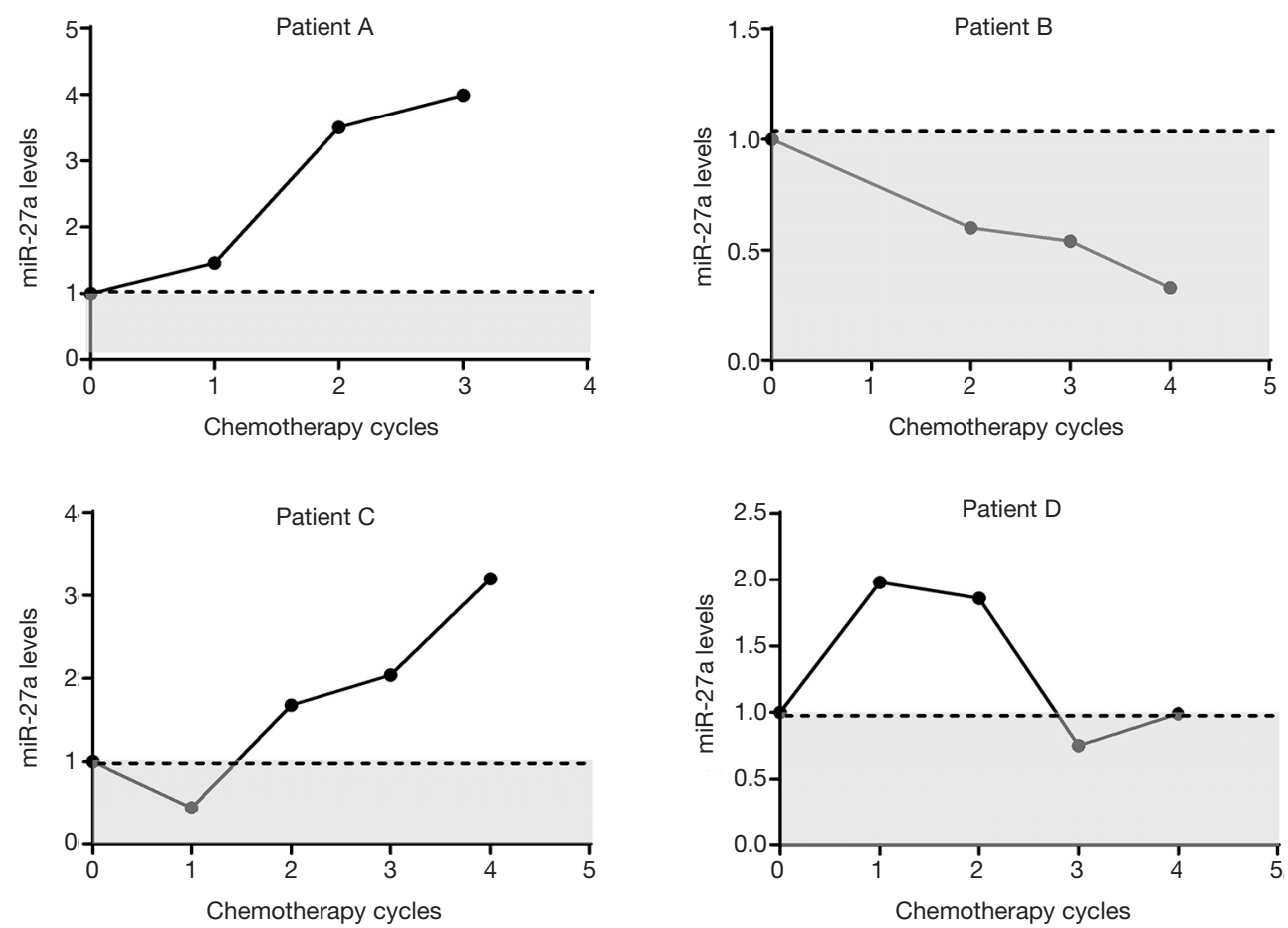

Figure 6 Dynamic changes in miR-27a levels at different time points following chemotherapy.

In a previous miRNA expression microarray analysis, we found that the expression levels of miR-27a in SPC-A1 cells treated with NJ001 increased in a time-dependent manner. To further discover the clinical application of miR27a in NSCLC, the apoptosis rates of SPC-A1 cells treated with $2.5 \mu \mathrm{g} / \mathrm{mL}$ cisplatin for different periods in vitro were measured. Simultaneously, miR-27a expression levels in culture supernatants and cells were measured. Our results suggested that SPC-A1 cell apoptosis was induced by cisplatin in a time-dependent manner, consistent with the research of Shi et al. (35). Importantly, the apoptosis rates were positively associated with the increased expression of miR-27a in a time-dependent manner in both SPC-A1 cells and culture supernatants. The expression of miR-27a increased with prolonged exposure and apoptosis rates, suggesting that miR-27a plays an active role in apoptosis.

The serum miR-27a levels of 52 patients with lung adenocarcinoma were also measured before and after chemotherapy by qRT-PCR analysis. We found that the proportion of patients with elevated serum miR-27a levels was $61.5 \%$ in the PR group and $30.8 \%$ in the NR group after the first chemotherapy cycle. This result suggested that increased serum miR-27a levels after chemotherapy were correlated with the prognosis of NSCLC patients.
Furthermore, Kaplan-Meier survival analysis showed that after first-line chemotherapy, the average survival times of patients with increased and decreased serum miR-27a levels were 28.4 vs. 9 months, respectively. These results suggested that patients with decreased miR-27a levels had poorer outcomes than those with increased miR-27a levels.

Previous research has shown that dynamic changes in miRNAs can be used to discriminate the type of response to antitumour therapy in lung cancer patients (36), and circulating tumour cells (CTCs) counts can be used for the dynamic monitoring and prediction of outcomes in patients with epidermal growth factor receptor (EGFR)mutated NSCLC treated with EGFR-tyrosine kinase inhibitors (TKIs) (37). Inspired by these studies, we evaluated the dynamic changes in the expression of miR$27 \mathrm{a}$ in the serum of representative patients before and after chemotherapy. The results showed that the level of serum miR-27a in the PR group increased gradually (patient A and patient $\mathrm{C}$ ), while that in the SD group and PD group decreased (patient B). Hence, these results revealed that dynamic changes in miR-27a levels in such individualized cases combined with therapeutic evaluations can be used to predict patient outcomes. A recent study has revealed that plasma level of miR-32 in NSCLC patients receiving 
platinum-based chemotherapy is considered as a prognostic indicator (38). Our findings showed that the elevated level of miR-27a in NSCLC patients receiving chemotherapy was able to predict the therapeutic efficacy of platinumbased chemotherapy and the prognosis. Nevertheless, a single biomarker may have limitations to predict the prognosis of NSCLC, and detecting a combination of prognostic biomarkers can be more reliable. We speculated that detecting both miR-27a miR-32 levels in patients with advanced NSCLC receiving chemotherapy is a promising approach to assist the evaluation of prognosis, which will be explored in future studies.

It is generally considered that genetic variation affects the chemotherapy response and overall survival of cancer patients. Because of gene variations, their therapeutic efficacy differs a lot in NSCLC patients receiving chemotherapy, with the chemotherapy response ranging from $26 \%$ to $60 \%$ (39). In particular, mutation subsets in NSCLC, including EGFR, anaplastic lymphoma kinase (ALK), c-ros oncogene 1 (ROS1) and v-raf murine viral oncogene homolog B1 (BRAF), are correlated with the sensitivity of chemotherapeutic agents or acquired resistance (40). Compared with these targets, miR-27a may serve as a promising marker for predicting the efficacy of chemotherapeutic agents and prognosis in NSCLC.

Liquid biopsy, which includes CTCs, circulating-free DNA (cfDNA), miRNAs, exosomes and tumour-educated platelets (TEPs), has potential use for non-invasive screening, early diagnosis, prognosis, response to treatment and real-time monitoring in NSCLC patients (41). Recently, many studies have demonstrated that a considerable number of miRNAs can be used as prognostic tools $(42,43)$. miR-27a is a well-identified miRNA in tumours, and its diverse functions depend mainly on the cancer type, as it plays a role in either cancer promotion or tumour suppression (44). In our study, there was a positive correlation between the expression of miR-27a and the apoptosis of tumour cells. Moreover, patients with increased miR-27a levels after the first cycle of chemotherapy had better outcomes than patients with decreased miR-27a levels. These results confirm that miR-27a may play a role in tumour suppression in lung cancer progression, consistent with the results of Acunzo et al. (30) but contradictory to those of Chae et al. (45). These contradictory results need to be confirmed by further research.

Currently, drug resistance remains the main cause of chemotherapeutic treatment failure (46). Relevant studies have identified that nucleotide excision repair (NSR) gene variation plays a key role in chemotherapeutic drug resistance, which reduces the efficacy of chemotherapeutic drugs through DNA damage repair $(47,48)$. Our findings have shown that miR27a levels can be used as a prognostic marker in NSCLC patients receiving chemotherapy. However, the mechanisms have not been fully revealed. Recent studies indicated that some miRNAs improve anticancer therapy by DNA damage repair (49). Therefore, we suspect that miR-27a can predict the sensitivity of chemotherapeutic drugs and the prognosis of patients by regulating NSR gene variation. For this, we will explore the relationship between miR-27a and NSR gene variation in NSCLC, which contributes to the individualized treatment of patients.

There are some limitations to be addressed in our study, such as a single-centre study with a small sample size. Therefore, the findings in this study will be investigated with multiple and large-scale samples in a wider population.

\section{Conclusions}

We found that the expression of miR-27a in the sera of NSCLC patients changed after the first chemotherapy cycle, and this change was related to the prognosis of NSCLC. More precisely, an increase in serum miR-27a is related to a good prognosis in NSCLC patients. Dynamic changes in serum miR-27a after each cycle of chemotherapy showing a gradual increase predict good clinical outcomes. Serum miR-27a may be a prognostic biomarker for NSCLC patients. However, the potential molecular mechanism of miR-27a in lung cancer and its therapeutic target for NSCLC need further study.

\section{Acknowledgments}

Funding: This study was supported by grants from Key Laboratory for Laboratory Medicine of Jiangsu Province of China (No. ZDXKB2016005), the National Natural Science Foundation of China $(81101322,81672100)$.

\section{Footnote}

Reporting Checklist: The authors have completed the STROBE reporting checklist. Available at http://dx.doi. org/10.21037/tcr-20-3276

Data Sharing Statement: Available at http://dx.doi. org/10.21037/tcr-20-3276 
Conflicts of Interest: All authors have completed the ICMJE uniform disclosure form (available at http://dx.doi. org/10.21037/tcr-20-3276). The authors have no conflicts of interest to declare.

Ethical Statement: The authors are accountable for all aspects of the work in ensuring that questions related to the accuracy or integrity of any part of the work are appropriately investigated and resolved. The study was conducted in accordance with the Declaration of Helsinki (as revised in 2013). The study was approved by the Ethics Committee of first affiliated hospital of Nanjing Medical University (No. 2015-SRFA-065, Nanjing, China) and informed consent was taken from all the patients.

Open Access Statement: This is an Open Access article distributed in accordance with the Creative Commons Attribution-NonCommercial-NoDerivs 4.0 International License (CC BY-NC-ND 4.0), which permits the noncommercial replication and distribution of the article with the strict proviso that no changes or edits are made and the original work is properly cited (including links to both the formal publication through the relevant DOI and the license). See: https://creativecommons.org/licenses/by-nc-nd/4.0/.

\section{References}

1. Miller KD, Nogueira L, Mariotto AB, et al. Cancer treatment and survivorship statistics, 2019. CA Cancer J Clin 2019;69:363-85.

2. Siegel RL, Miller KD, Jemal A. Cancer statistics, 2019. CA Cancer J Clin 2019;69:7-34.

3. Altorki NK, Kamel MK, Narula N, et al. Anatomical segmentectomy and wedge resections are associated with comparable outcomes for patients with small cT1N0 nonsmall cell lung cancer. J Thorac Oncol 2016;11:1984-92.

4. Tabchi S, Kassouf E, Rassy EE, et al. Management of stage III non-small cell lung cancer. Semin Oncol 2017;44:163-77.

5. Hotta K, Kiura K, Fujiwara Y, et al. Role of survival postprogression in phase III trials of systemic chemotherapy in advanced non-small-cell lung cancer: a systematic review. PLoS One 2011;6:e26646.

6. Zhang J, Raju GS, Chang DW, et al. Global and targeted circulating microRNA profiling of colorectal adenoma and colorectal cancer. Cancer 2018;124:785-96.

7. Bhaskaran M, Mohan M. MicroRNAs: history, biogenesis, and their evolving role in animal development and disease.
Vet Pathol 2014;51:759-74.

8. Wang J, Liu S, Li J, et al. Roles for miRNAs in osteogenic differentiation of bone marrow mesenchymal stem cells. Stem Cell Res Ther 2019;10:197.

9. Wang M, Liu C, Su Y, et al. miRNA-34c inhibits myoblasts proliferation by targeting YY1. Cell Cycle 2017;16:1661-72.

10. Song YS, Joo HW, Park IH, et al. Bone marrow mesenchymal stem cell-derived vascular endothelial growth factor attenuates cardiac apoptosis via regulation of cardiac miRNA-23a and miRNA-92a in a rat model of myocardial infarction. PLoS One 2017;12:e0179972.

11. Pan S, Wang F, Huang $P$, et al. The study on newly developed McAb NJ001 specific to non-small cell lung cancer and its biological characteristics. PLoS One 2012;7:e33009.

12. Wang F, Lou JF, Cao Y, et al. miR-638 is a new biomarker for outcome prediction of non-small cell lung cancer patients receiving chemotherapy. Exp Mol Med 2015;47:e162.

13. Li X, Xu M, Ding L, et al. MiR-27a: a novel biomarker and potential therapeutic target in tumors. J Cancer 2019;10:2836-48.

14. Li Y, Li J, Sun X, et al. MicroRNA-27a functions as a tumor suppressor in renal cell carcinoma by targeting epidermal growth factor receptor. Oncol Lett 2016;11:4217-23.

15. Bao Y, Chen Z, Guo Y, et al. Tumor suppressor microRNA-27a in colorectal carcinogenesis and progression by targeting SGPP1 and Smad2. PLoS One 2014;9:e105991.

16. Wang YL, Gong WG, Yuan QL. Effects of miR-27a upregulation on thyroid cancer cells migration, invasion, and angiogenesis. Genet Mol Res 2016. doi: 10.4238/ gmr15049070.

17. Relli V, Trerotola M, Guerra E, et al. Abandoning the Notion of Non-Small Cell Lung Cancer. Trends Mol Med 2019;25:585-94.

18. Choe G, Carr R, Molena D. New surgical approaches in the treatment of non-small cell lung cancer. Clin Chest Med 2020;41:175-83.

19. Bartel DP. MicroRNAs: genomics, biogenesis, mechanism, and function. Cell 2004;116:281-97.

20. Zhang Y, Sui J, Shen X, et al. Differential expression profiles of microRNAs as potential biomarkers for the early diagnosis of lung cancer. Oncol Rep 2017;37:3543-53.

21. Qi L, Gao C, Feng F, et al. MicroRNAs associated with lung squamous cell carcinoma: new prognostic 
biomarkers and therapeutic targets. J Cell Biochem 2019;120:18956-66.

22. Yuwen DL, Sheng BB, Liu J, et al. MiR-146a-5p level in serum exosomes predicts therapeutic effect of cisplatin in non-small cell lung cancer. Eur Rev Med Pharmacol Sci 2017;21:2650-8.

23. Deng H, Qianqian G, Ting J, et al. miR-539 enhances chemosensitivity to cisplatin in non-small cell lung cancer by targeting DCLK1. Biomed Pharmacother 2018;106:1072-81.

24. Filipska M, Skrzypski M, Czetyrbok K, et al. MiR-192 and miR-662 enhance chemoresistance and invasiveness of squamous cell lung carcinoma. Lung Cancer 2018;118:111-8.

25. Mehta A, Dobersch S, Romero-Olmedo AJ, et al. Epigenetics in lung cancer diagnosis and therapy. Cancer Metastasis Rev 2015;34:229-41.

26. Hashemi ZS, Khalili S, Forouzandeh Moghadam M, et al. Lung cancer and miRNAs: a possible remedy for antimetastatic, therapeutic and diagnostic applications. Expert Rev Respir Med 2017;11:147-57.

27. Pan J, Zhou C, Zhao X, et al. A two-miRNA signature (miR-33a-5p and miR-128-3p) in whole blood as potential biomarker for early diagnosis of lung cancer. Sci Rep 2018;8:16699.

28. Yang M, Shen H, Qiu C, et al. High expression of miR21 and miR-155 predicts recurrence and unfavourable survival in non-small cell lung cancer. Eur J Cancer 2013;49:604-15.

29. Li S, Li J, Fei BY, et al. MiR-27a promotes hepatocellular carcinoma cell proliferation through suppression of its target gene peroxisome proliferator-activated receptor $\gamma$. Chin Med J 2015;128:941-7.

30. Acunzo M, Romano G, Palmieri D, et al. Cross-talk between MET and EGFR in non-small cell lung cancer involves miR-27a and Sprouty2. Proc Natl Acad Sci USA 2013;110:8573-8.

31. Fletcher CE, Dart DA, Sita-Lumsden A, et al. Androgenregulated processing of the oncomir miR-27a, which targets Prohibitin in prostate cancer. Hum Mol Genet 2012;21:3112-27.

32. Drayton RM, Dudziec E, Peter S, et al. Reduced expression of miRNA-27a modulates cisplatin resistance in bladder cancer by targeting the cystine/glutamate exchanger SLC7A11. Clin Cancer Res 2014;20:1990-2000.

33. Zhao X, Yang L, Hu J. Down-regulation of miR-27a might inhibit proliferation and drug resistance of gastric cancer cells. J Exp Clin Cancer Res 2011;30:55.
34. Wu XZ, Wang KP, Song HJ, et al. MiR-27a-3p promotes esophageal cancer cell proliferation via F-box and WD repeat domain-containing 7 (FBXW7) suppression. Int J Clin Exp Med 2015;8:15556-62.

35. Shi L, Xu Z, Wu G, et al. Up-regulation of miR-146a increases the sensitivity of non-small cell lung cancer to DDP by downregulating cyclin J. BMC Cancer 2017;17:138.

36. Ponomaryova AA, Morozkin ES, Rykova EY, et al. Dynamic changes in circulating miRNA levels in response to antitumor therapy of lung cancer. Exp Lung Res 2016;42:95-102.

37. Jiang T, Zhao J, Zhao C, et al. Dynamic monitoring and predictive value of circulating tumor cells in EGFRmutated advanced non-small-cell lung cancer patients treated with first-line EGFR tyrosine kinase inhibitors. Clin Lung Cancer 2019;20:124-33.e2.

38. Xu S, Li J, Chen L, et al. Plasma miR-32 levels in nonsmall cell lung cancer patients receiving platinum-based chemotherapy can predict the effectiveness and prognosis of chemotherapy. Medicine (Baltimore) 2019;98:e17335.

39. Bahl A, Falk S. Meta-analysis of single agents in the chemotherapy of NSCLC: what do we want to know? Br J Cancer 2001;84:1143-5.

40. Doroshow DB, Herbst RS. Treatment of advanced non-small cell lung cancer in 2018. JAMA Oncol 2018;4:569-70.

41. Pérez-Callejo D, Romero A, Provencio M, et al. Liquid biopsy based biomarkers in non-small cell lung cancer for diagnosis and treatment monitoring. Transl Lung Cancer Res 2016;5:455-65.

42. Nagaraj AB, Joseph P, DiFeo A. miRNAs as prognostic and therapeutic tools in epithelial ovarian cancer. Biomark Med 2015;9:241-57.

43. Yu C, Wan H, Shan R, et al. The prognostic value of the miR-200 family in colorectal cancer: a meta-analysis with 1882 patients. J Cancer 2019;10:4009-16.

44. Tian Y, Fu S, Qiu GB, et al. MicroRNA-27a promotes proliferation and suppresses apoptosis by targeting PLK2 in laryngeal carcinoma. BMC Cancer 2014;14:678.

45. Chae DK, Ban E, Yoo YS, et al. MIR-27a regulates the TGF- $\beta$ signaling pathway by targeting SMAD2 and SMAD4 in lung cancer. Mol Carcinog 2017;56:1992-8.

46. Zhitomirsky B, Assaraf YG. Lysosomes as mediators of drug resistance in cancer. Drug Resist Updat 2016;24:23-33.

47. Li D, Zhou Q, Liu Y, et al. DNA repair gene polymorphism associated with sensitivity of lung cancer to 
therapy. Med Oncol 2012;29:1622-8.

48. Song X, Wang S, Hong X, et al. Single nucleotide polymorphisms of nucleotide excision repair pathway are significantly associated with outcomes of platinum-based chemotherapy in lung cancer. Sci Rep 2017;7:11785.

49. Bottai G, Pasculli B, Calin G, et al. Targeting the microRNA-regulating DNA damage/repair pathways in cancer. Expert Opin Biol Ther 2014 14:1667-83.

Cite this article as: Xie E, Lin M, Sun Z, Jin Y, Zhang S, Huang L, Sun R, Wang F, Pan S. Serum miR-27a is a biomarker for the prognosis of non-small cell lung cancer patients receiving chemotherapy. Transl Cancer Res 2021;10(7):3458-3469. doi: $10.21037 /$ tcr-20-3276 